\title{
Frontières
}

\section{Des casques bleus démunis face à un génocide en Bosnie-Herzégovine}

\section{La mort et le sacrifice ultime}

\section{Michel Sartori}

Volume 14, numéro 2, printemps 2002

La mort prononcée

URI : https://id.erudit.org/iderudit/1073977ar

DOI : https://doi.org/10.7202/1073977ar

Aller au sommaire du numéro

Éditeur(s)

Université du Québec à Montréal

ISSN

1180-3479 (imprimé)

1916-0976 (numérique)

Découvrir la revue

Citer ce document

Sartori, M. (2002). Des casques bleus démunis face à un génocide en

Bosnie-Herzégovine : la mort et le sacrifice ultime. Frontières, 14(2), 78-81.

https://doi.org/10.7202/1073977ar d'utilisation que vous pouvez consulter en ligne.

https://apropos.erudit.org/fr/usagers/politique-dutilisation/ 


\section{Des casques bleus démunis face à un génocide en Bosnie-Herzégovine La mort et le sacrifice ultime}

\author{
Michel Sartori, \\ capitaine, Armée canadienne.
}

Pour rédiger cet article, j'ai choisi de m'inspirer de mon expérience comme militaire, de rencontres et d'études touchant divers aspects de la vie du soldat membre des Forces armées canadiennes. Ma réflexion porte sur le rôle du soldat en mission de maintien de la paix des Nations Unies à travers le monde, la principale difficulté étant alors de se retrouver brutalement confronté à sa propre mort à travers la mort des autres, en voyant sa propre vie menacée. Celui qui n'a jamais vu la mort de près auparavant ne sait pas se situer face à celle-ci. S'il est croyant, il n'a entendu parler que de l'au-delà, comme seule réponse au mystère de la mort. Dans la plupart des cas, le soldat est confronté à la mort sans y avoir consacré, auparavant, une réflexion vraiment profonde. D'abord parce qu'il est à un âge où sa préoccupation première ne porte pas sur le sens à donner à sa propre mort. En fait, il n'a pas été préparé à cette réalité!

Sur le plan socioculturel, la formation est également insuffisante; le soldat découvre sur le terrain les coutumes de peuples de diverses croyances et peut alors se trouver totalement démuni pour intervenir de façon appropriée. Les conflits inter- ethniques et les luttes alimentées par des nationalistes intransigeants sont de plus en plus nombreux et violents à travers le monde, mais inconnus chez nous. Cela rend les opérations militaires complexes et difficiles sur le plan psychologique. Le casque bleu est coincé au centre de combats passionnés et quasi impossibles à contrôler. Il doit assister, impuissant, à des meurtres et à des séances de torture sans pouvoir réagir, étant donné que son mandat ne l'autorise pas à intervenir par les armes. Ce qui m'amène à témoigner des difficultés auxquelles le soldat doit faire face en mission de maintien de la paix, et de l'importance d'une formation adéquate, car il sera confronté à la mort et à la torture des civils par les belligérants, ainsi qu'à l'éventualité de sa propre mort. Une préparation à sa souffrance psychologique est aussi essentielle afin d'éviter les nombreux cas de maladies post-traumatiques au retour de missions. À cet égard, je formule ici certaines critiques à l'endroit des autorités militaires qui semblent avoir préféré éluder la question plutôt que de faire appel à des spécialistes pour une meilleure préparation des soldats.

Il convient aussi de souligner l'isolement dans lequel se retrouve le soldat en rentrant au pays. On ne lui manifeste pas la moindre reconnaissance à l'égard de son dévoue- ment et des sacrifices personnels qu'il a consentis. Non seulement il n'est pas vraiment accueilli, mais aussitôt arrivé, il doit reprendre les exercices pour une autre mission. La politique du délai de 18 mois entre les missions n'est pas respectée. Les fréquents changements de commandement rendent aussi la tâche plus difficile; les officiers supérieurs n'ont pas le temps de se préparer.

\section{LES DROITS DE LA PERSONNE}

On peut aborder le sujet en commençant par les grandes résolutions de maintien de la paix à travers les nations, formulées en des termes qui ne prêtent à aucune équivoque. Je veux citer ici un texte fort et explicite : l'article 1 de la Charte des Nations Unies, adoptée à la suite de la Seconde Guerre mondiale :

Nous, Peuple des Nations Unies, résolus $[. .$.$] à préserver les générations$ futures du fléau de la guerre [...] à créer les conditions nécessaires au maintien de la justice [...] à proclamer de nouveau notre foi dans les droits fondamentaux de la personne [...] à préserver la liberté et l'autonomie de chaque peuple $[\ldots]$.

Cette volonté de maintenir la paix et de réaffirmer cette foi dans les droits fonda- 
mentaux de la personne contraste entre l'idéal et la dure réalité. Car, dans les faits, les buts poursuivis par les Nations Unies n'ont pas suffi à faire cesser les crimes atroces qui ont continué de se répandre dans l'ancienne République de Yougoslavie, au Rwanda, en Afrique et ailleurs dans le monde. Accentuant cette tragédie, les crimes que l'humanité considérait comme des démons du passé, des coins obscurs de l'histoire humaine ont pour théâtre le cœur de la vieille Europe politisée et ordonnée, et pour public, l'opinion internationale.

Il convient de se demander quelle a été la portée réelle de cette Déclaration, quant à la liberté et à l'autonomie des peuples, lorsque l'on constate que des atrocités se poursuivent toujours dans certains pays. En voulant préserver cette liberté, aurait-on encouragé la violence sous tous les prétextes? Pour justifier certaines revendications plus ou moins légitimes, on continue de tuer et de torturer des innocents, alors que nous restons impassibles devant ces horreurs qui se multiplient sous les regards du monde entier! Les appels à la paix et à la tolérance ne font qu'alimenter l'antagonisme et la domination envers les plus démunis. Cette réalité est bien loin du maintien de la justice et des droits fondamentaux des hommes, évoqués dans l'imposant document des Nations Unies! En prenant de telles résolutions, suffisait-il de s'appuyer sur la présence des casques bleus, déployés dans chaque coin du monde, pour empêcher que des nations s'affrontent? Comment quelques soldats peuvent-ils réussir à maintenir la paix face à des belligérants qui en déploient des centaines? Le mandat des casques bleus en mission de paix est clair: ils ne doivent en aucun cas intervenir militairement; ils ne sont armés que pour intimider l'adversaire, mais il leur est interdit d'utiliser leurs armes pour protéger le peuple dont on leur a confié la responsabilité, ni pour se protéger eux-mêmes.

Militaire depuis 14 ans, j'ai assisté à des atrocités lors de plusieurs missions de paix des Nations Unies, comme membre de deux importantes formations dont le célèbre régiment d'élite, le régiment aéroporté du Canada, qu'on a surnommé les commandos, et du Royal 22e Régiment. Ma dernière mission fut celle de Bosnie-Herzégovine, au cours de laquelle j'ai été témoin de violentes manifestations qui m'ont profondément bouleversé. C'est par ce génocide que j'ai pris conscience que les êtres humains peuvent se laisser emporter par des instincts primitifs et perdre toute capacité de distinguer le bien du mal. Cette expérience m'a aussi permis de connaître mes limites et même de les dépasser.

Il importe de rappeler ici qu'en Bosnie, comme au Rwanda d'ailleurs, notre rôle se limitait par définition à protéger la population. Alors que les Serbes défiaient le monde entier, les pays européens, les Nations Unies et les États-Unis semblaient incapables de faire cesser les massacres. Limitant notre mission à rapporter à nos supérieurs ce qui se passait sur le terrain, les Nations Unies étaient, en quelque sorte, spectateurs de ce génocide. Ainsi, notre mission de maintenir la paix sans réagir perdait tout son sens.

Le fait d'assister, impuissants, à la mort de nombreux innocents créait la plus grande frustration chez les soldats. Il devenait de plus en plus difficile d'obéir aux ordres de nos supérieurs, malgré les risques qu'une telle désobéissance comporte. Alors que des personnes étaient tuées sous nos nelle. Le sens du devoir et de la responsabilité sociale chez les militaires qui occupent des positions de commandement fait partie intrinsèque des règles de l'éthos militaire. Conséquemment, le militaire canadien, à qui l'on confie sous certaines formes la sécurité de la nation et le lourd mandat de gardien de la paix, peut se voir obligé de faire le sacrifice ultime au nom de son pays et des droits fondamentaux de la personne ${ }^{1}$.

En dépit de l'adhésion à l'éthos militaire, d'autres questions méritent d'être posées. Lorsqu'il est en mission, le militaire canadien est-il prêt à mourir ? Est-il préparé psychologiquement à mourir ? Doit-il accepter d'affronter la mort à tout instant pour n'importe quelle cause? À ces questions,

\section{DÉCLARER LA GUERRE, C'EST EN QUELQUE SORTE ANNONCER}

À L'ENNEMI QU'IL VA MOURIR. POUR LE SOLDAT, C'EST PRESQUE TOUJOURS DE LA MORT DES AUTRES

DONT IL EST QUESTION.

yeux et que le viol était systématiquement utilisé comme instrument de guerre, nous devions demeurer impassibles devant ces atrocités pour respecter le rôle qui nous était assigné. Et que dire du peu de moyens que nous possédions pour protéger les membres de notre section contre les attaques ennemies: cela se limitait à notre débrouillardise. Ces missions sont dures, surtout au point de vue psychologique parce que leur objectif n'est pas clair. Il convient aussi de souligner ici le manque de soutien logistique militaire et d'appui moral de la part des autorités.

Plus encore, que penser des positions morales et politiques de nos décideurs qui étaient au fait de ces crimes innommables? À cet égard, la Communauté internationale refuse de se rendre à l'évidence que sa politique prétendant maintenir la paix et préserver les droits fondamentaux de la personne est un échec. Les droits humains ne semblent représenter que des mots vides de sens; certains êtres humains ne distinguent plus le bien du mal et l'absence de respect et d'amour pour l'humanité mène tout droit à la destruction de la planète.

\section{LE MORT ANNONCÉE}

Déclarer la guerre, c'est en quelque sorte annoncer à l'ennemi qu'il va mourir. Pour le soldat, c'est presque toujours de la mort des autres dont il est question. Bien sûr, l'Armée canadienne nous prépare à faire face à l'ennemi dans une guerre convention- la réponse est non! La doctrine militaire nous enseigne diverses stratégies pour détruire et tuer l'ennemi, mais ne fait aucune référence à notre propre mort. $\mathrm{Ce}$ constat est étonnant, car il sous-entend l'invincibilité du soldat et perpétue le mythe du superhéros. Ce « lavage de cerveau » ou, pour employer un terme plus militaire, cet endoctrinement, n'a pour objectif que de nous empêcher de penser à notre propre mort, car en situation de guerre cela pourrait paralyser la capacité d'action du soldat et être interprété comme un signe de faiblesse. Pourtant, entre 1992 et 1994, il y a eu chez les militaires canadiens en exYougoslavie 23 morts, 300 blessés (incluant les blessures par balles, par éclats d'obus, par mines, amputations de pieds et de jambes) ${ }^{2}$. De plus, environ un Casque bleu canadien sur six souffre de problèmes psychologiques sérieux depuis son retour de l'ex-Yougoslavie. Près de $15 \%$ des soldats canadiens qui ont servi pendant cette période souffrent de stress post-traumatique et environ $13 \%$ de dépression ${ }^{3}$. Finalement, 50 suicides ou tentatives de suicide par semaine chez les militaires canadiens font partie de la réalité observée aujourd'hui ${ }^{4}$.

Face à ce constat, le mythe du superhéros en prend un coup. Le militaire, pour préserver son image d'invincibilité, s'enfonce un peu plus dans l'isolement par peur d'être ridiculisé si ses supérieurs apprenaient qu'il est en détresse psychologique. Il n'a pas été formé à s'apitoyer sur ses 
propres souffrances et encore moins à en parler. En fait, il n'a pas le droit de connaître et d'exprimer ses sentiments comme tout être humain normal: l'angoisse, la frayeur et l'appréhension. Pourtant, c'est ce qu'il vit à chaque instant du jour et de la nuit durant de tels conflits armés, même s'il est prêt à tuer au nom de la paix. C'est le sentiment que j'ai ressenti à mon arrivée à Sarajevo, mais j'ai tôt fait de me rendre compte de mon impuissance face à cette purification ethnique. Je n'avais que mon drapeau bleu des Nations Unies flottant sur mon véhicule blindé et une veste antifragmentation; c'était peu efficace contre les tireurs d'élite, dont nous étions les cibles.

L'absence de règles d'engagement claires, le manque d'expérience de certains sousofficiers et officiers à des postes clés et l'absence de préparation psychologique ont eu bien souvent raison des vaillants soldats. La force physique et le courage ne suffisaient pas toujours à surmonter les obstacles. En ce qui me concerne, bien que j'aie déjà participé à quelques exercices de camp de prisonniers (maintenant interdits dans l'Armée canadienne), ces exercices, qui avaient pour objectif de préparer le soldat à supporter les sévices psychologiques et physiques, étaient bien loin de la réalité. Je ne crois pas que cela m'ait été vraiment utile lorsque j'ai été à quatre reprises pris en otage, alors que j'ai dû négocier personnel- lement la libération de 16 soldats canadiens retenus en territoire serbe. Cette libération a été rendue possible au péril de nos vies, ma section et moi ayant servi de rançon. Ce geste est pourtant passé sous silence, nos supérieurs étant trop occupés à médiatiser la libération de ces 16 Canadiens. Aussi incroyable que cela puisse paraître, en aucun cas il n'a été fait mention que nous nous étions sacrifiés pour calmer l'opinion publique au Canada.

Durant ces moments graves, nous avons bien sûr envisagé notre propre mort, mais tous nos efforts visaient avant tout à $\mathrm{y}$ échapper, pour cette fois du moins. Cette même situation s'est présentée à deux autres reprises. La première fois, nous venions de prendre position à un poste d'observation, lorsque le commandant du secteur serbe est venu m'ordonner de quitter les lieux immédiatement, sans quoi nous serions tous tués dans moins de deux heures. Je me suis dit alors: «Mon Dieu! Pas encore une fois! » J'ai informé par téléphone mon quartier général de ces menaces et tout ce que j'ai obtenu comme réponse, c'est: "Vous devez défendre votre position à tout prix. » Je me suis dit, ce n'est pas sérieux, nous sommes isolés en territoire serbe et entourés de milliers de belligérants. Puis, un deuxième avertissement de quitter les lieux de la part des opposants, sous peine de tous être tués, nous a bien fait comprendre qu'il fallait nous préparer sérieusement à faire face à la menace. J'ai donc informé de nouveau mon quartier général de la détermination des belligérants de passer à l'action si nous refusions de quitter les lieux sur-le-champ. Cette fois, le message a été bref: "Attendez! ... terminé...». La communication était interrompue. Ce qui vous renvoie tout simplement la responsabilité. Il me fallait donc agir vite et user de stratégies prudentes en songeant à tous ceux que je devais protéger. Devant l'indifférence de mes supérieurs, et ne voulant pas risquer la vie des neuf membres de ma section, j'ai pris l'initiative de déplacer mon poste d'observation de 50 mètres, ce qui a satisfait nos opposants. Cette décision me semblait la plus logique si je voulais repousser le moment fatidique, car je n'étais pas prêt à faire le sacrifice ultime pour un bout de terrain qui s'est révélé par la suite la juste propriété des Serbes. À cette occasion, mes hommes et moi avons réalisé que nous n'étions que de la chair à canon pour nos supérieurs, car si nous avions obéi à l'ordre reçu, nous aurions tous été tués. Mais qu'est-ce qu'un tel risque représente pour un général qui est à des milliers de mètres de la réalité ?

Un mois plus tard, nous avons reçu l'ordre de nous déployer à Bakovici. Notre mission était de protéger les patients de cet hôpital psychiatrique qui avaient été laissés à eux-mêmes, le personnel ayant quitté en

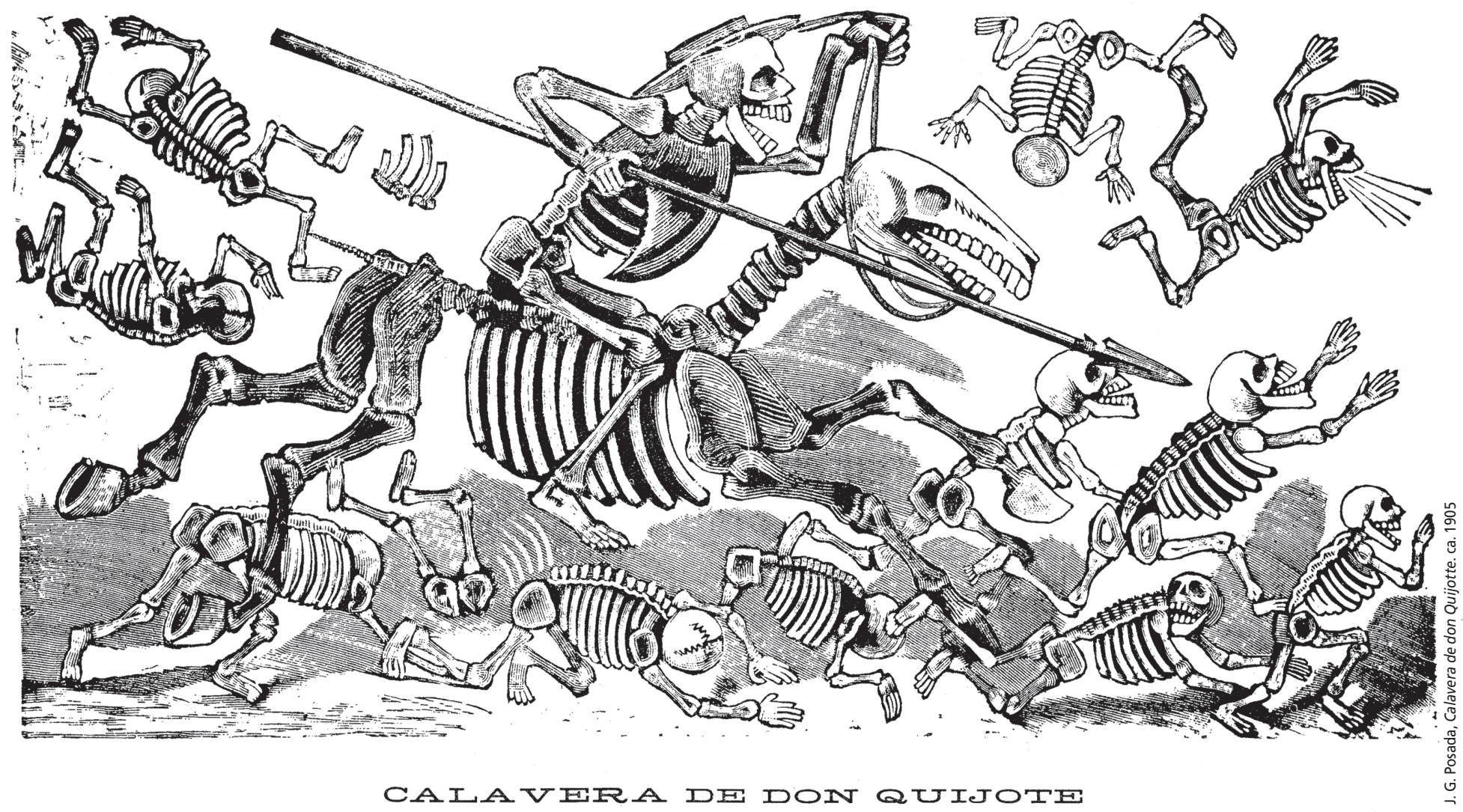


raison des attaques de l'ennemi dirigées vers l'hôpital. Ces malades, tout à fait démunis, étaient devenus des cibles faciles pour les belligérants qui s'adonnaient à des actes innommables sur ces innocentes victimes: on entrait, violait et tuait sans distinction d'âge ces patients sans défense. À notre arrivée, mes supérieurs m'ont avisé par radio qu'une attaque contre notre position aurait lieu dans les trois jours suivants et que je devais défendre l'hôpital psychiatrique de Bakovici. Je n'ai pas immédiatement pris la chose au sérieux, car nous étions 10 contre peut-être 800 . J'ai tout de même demandé qu'on m'envoie du personnel en renfort de même que des troupes blindées. Mais une fois de plus, nous devions trouver nous-mêmes la solution, car la réponse de mes supérieurs fut négative. Malgré mes doutes sur la véracité de cette information, j'ai demandé qu'on me fournisse d'urgence une caisse de grenades, une caisse de M72 (armes anti-char) ainsi que des munitions supplémentaires 5,56 mm. À ma grande surprise, ces munitions m'ont été livrées dès le lendemain matin. J'ai alors pris conscience que j'aurais à assumer d'énormes responsabilités dans les 72 heures. Je dois avouer que j'étais pris de frayeur, mais je ne devais pas m'y arrêter; il me fallait réagir rapidement. Comme on m'avait refusé du personnel, je me suis mis à la tâche en préparant un plan de défense. J'ai d'abord informé les membres de ma section d'une attaque imminente ainsi que du refus essuyé lors de ma demande de renforts auprès de notre quartier général. Certains se sont mis à pleurer, d'autres manifestaient des signes évidents d'extrême nervosité. Quant à moi, j'arrivais difficilement à contenir ma peur, mais j'étais conscient que je devais calmer l'anxiété de mes soldats en adoptant une attitude impassible et un comportement rassurant face à la situation. Déjà, nos adversaires étaient aux portes de l'hôpital et nous indiquaient clairement par leurs gestes que nous allions tous mourir, ce qui n'était pas pour remonter le moral de mes hommes. L'heure était tragique et nous avions besoin de réconfort. C'est la visite du prêtre qui nous a soutenus moralement; il a administré aux soldats le sacrement du pardon. Ce moment fut capital pour ma section; mes hommes ont repris confiance en eux et disaient être prêts au sacrifice ultime pour accomplir notre mission. Comme nous l'avait annoncé, l'attaque s'est produite, mais les belligérants n'ont pu pénétrer à l'intérieur des murs de l'hôpital. Grâce à notre présence déterminée et comme nous étions mieux équipés en munitions, nous avons réussi à les impressionner. En faisant étalage de nos armes, notre but était, bien sûr, de les intimider. Il n'en demeure pas moins que cette épreuve m'a ébranlé : j'ai dû être transporté d'urgence à l'hôpital de Visoko pour des douleurs à la poitrine, les médecins craignant le début d'une attaque cardiaque. Je suis demeuré deux jours à l'hôpital, puis j'ai demandé au médecin la permission de retourner à mon poste, en promettant de surveiller mes limites.

\section{MOURIR SUR DEMANDE... DE QUI?}

Les deux situations qui viennent d'être décrites laisseront peut-être croire au lecteur que je serais prêt à mourir pour une cause qui me semblerait juste. L'exemple des patients de l'hôpital de Bakovici en serait une. Par ailleurs, malgré mon amour inconditionnel envers l'être humain, il y a de fortes chances que je refuse l'ordre d'un supérieur qui ne serait pas au poste de commande, mais assis confortablement dans les bureaux de son quartier général. Pensons à notre histoire militaire où de nombreux Canadiens et Alliés se sont fait tuer uniquement pour satisfaire la pure folie de certains dirigeants. Dans ces conditions, non, je ne serais pas prêt à me faire tuer. Car au nom du patriotisme, combien de soldats ont dû sacrifier leur vie, forcés de respecter un plan qui, très souvent, n'avait aucune chance de réussir? J'éprouve un immense respect pour ceux qui ont donné leur vie dans l'espoir de nous assurer la liberté. Néanmoins, je me suis souvent demandé combien valait la vie d'un soldat si l'on constate que $10 \%$ en pertes de vies humaines semble une norme acceptable pour un général, alors que l'élément le plus précieux pour une armée, c'est le soldat.

En conclusion, cette mission de paix en Bosnie a été une expérience traumatisante dont plusieurs sont revenus réellement bouleversés. Entre autres, parce que nous n'étions pas préparés à faire face à ce genre de conflit. Le manque d'expérience de nos officiers, l'absence quasi totale de ressources et un mandat qui ne correspondait pas au rôle de gardiens de la paix face à une armée dont le rapport de forces était totalement déséquilibré. En fait, on nous demandait de servir de tampon pour protéger les civils contre les attaques des belligérants, dont l'objectif était de répandre le maximum de terreur dans la population civile, d'exercer le maximum de violence sur des femmes et des enfants, alors que notre seule stratégie se limitait à tenter de les intimider. Nous étions donc constamment confrontés à notre propre mort sans y avoir été préparés psychologiquement. En effet, notre formation militaire n'a aucun point commun avec une guerre dont les instruments sont le viol et le massacre de femmes et d'enfants innocents et auxquels nous assistions impuissants à réagir, faute de moyens appropriés! Dans un tel contexte, nous étions effrayés non seulement par la mort des autres, mais aussi par l'éventualité de notre propre mort. La peur de devoir affronter le sacrifice ultime nous envahissait à tel point qu'il ne nous venait pas à l'esprit de nous y préparer sérieusement! Pour ma part, j'aurais été prêt à faire ce sacrifice ultime, mais à la condition d'être inspiré par un leadership sur le terrain et d'être assuré d'un soutien moral et financier pour ma famille. Toutefois, l'expérience vécue à mon retour de mission ne m'inspire aucun espoir en ce sens! Enfin, je croirais à un partage équitable des risques sur le terrain à tous les niveaux de la chaîne de commandement.

\section{Notes}

1. Éthique et conscience professionnelle, École des aspirants officiers, B.F.C. de Chiliwack.

2. «Les opérations dans l'ancienne République de Yougoslavie", Journal de l'infanterie, $\mathrm{n}^{\circ} 27$, vol. $4, \mathrm{n}^{\circ} 1$, septembre 1996 .

3. Loi sur l'accès à l'information, étude du lieutenant-commander Passey, psychiatre militaire, concernant les troubles du stress post-traumatique des militaires, QGCFT, Ottawa, 1992-1994.

4. Rencontre des sous-officiers avec l'adjudantchef des Forces canadiennes (Maurice Dessureault), tenue au mess du $51^{\mathrm{e}} \mathrm{BNS} d \mathrm{u}$ Canada, décembre 2000. 